\title{
INTERNAL ROTATION AND DYNAMICS OF THE SUN FROM GONG DATA
}

\author{
S. G. KORZENNIK
}

Harvard-Smithsonian Center for Astrophysics

60 Garden St, Cambridge MA 02138, USA

M. J. THOMPSON

Astronomy Unit, Queen Mary $\&$ Westfield College

Mile End Rd, London E1 $4 N S$, UK

J. TOOMRE

Joint Institute for Laboratory Astrophysics

University of Colorado, Boulder CO 80309, USA

AND

THE GONG INTERNAL ROTATION TEAM

\section{Introduction}

We report inferences for the Sun's internal rotation from GONG months 4-10 averaged power spectra. ${ }^{1}$ In keeping with the international collaborative nature of the GONG project, the results presented here are based on the work of several groups around the world inverting the GONG data and sharing their results via the world-wide web. These groups are at the $\mathrm{Ob}$ servatoire de la Côte d'Azur, Nice (T. Corbard, G. Berthomieu, J. Provost); Theoretical Astrophysics Center, Aarhus (J. Christensen-Dalsgaard, F. Pijpers); Center for Astrophysics, Cambridge MA (A. Eff-Darwich, S. Korzennik); QMW, London (R. Howe, M. Thompson, in collaboration with J. Schou, Stanford); Institute of Astronomy, Cambridge (T. Sekii, D. Gough);

${ }^{1}$ This work utilizes data obtained by the Global Oscillation Network Group (GONG) project, managed by the National Solar Observatory, a Division of the National Optical Astronomy Observatories, which is operated by AURA, Inc. under a cooperative agreement with the National Science Foundation. The data were acquired by instruments operated by the Big Bear Solar Observatory, High Altitude Obseratory, Learmonth Solor Observatory, Udaipur Solor Observatory, Instituto de Astrofísico de Canarias, and Cerro Tololo Interamerican Observatory. 
University of Sydney (D. Burtonclay, Li Yan, P. Wilson); and Tata Institute of Fundamental Research, Bombay (H. Antia, S. Chitre).

\section{Key issues}

The first inferences made by the team from GONG data were presented in the special GONG issue of Science (Thompson et al. 1996). The present paper is a report on work in progress towards a more mature understanding of the inferences that can be drawn from the GONG data at the present time. Two principal datasets have been used, both based on averaged 7month (GONG months 4-10) power spectra. One set comprises individual $m$ frequencies from the GONG project pipeline (Hill et al. 1996), in the ranges $\nu<5000 \mu \mathrm{Hz}$ and $0 \leq l \leq 150$ : this set contains 109483 frequencies with IERR and BAD flags ${ }^{2}$ both zero, though individual inverters will generally have made smaller selections and/or fitted low-order polynomials in $m$ to these frequencies. The second set consists of 7910 Clebsch-Gordon $a$ coefficients in approximately the same ranges of frequency and degree (1111 $\mu \mathrm{Hz} \leq \nu \leq 4563 \mu \mathrm{Hz}, 5 \leq l \leq 150)$, obtained by S. Korzennik using a completely different frequency estimation procedure.

In addition to the global picture of the internal rotation of the Sun, the particular aspects of the solar rotation that we have identified as likely targets for study with these data are:

- near-surface shear layer

- shear layer at the base of the convection zone (tachocline)

- variations within the convection zone

- latitudinal constancy in the radiative interior

- rotation of the core

Another important issue that must be addressed is the reliability of the inferences that we make, and the sources of uncertainty. Thus we wish to assess:

- uncertainties coming from the data reduction (peak finding)

- discrepancies from inverting different observations

- discrepancies between different inversions of the same data

Then we might hope to give an answer to the question posed by John Leibacher during this conference: "How can we render more certain our inferences from the data?".

\footnotetext{
${ }^{2}$ IERR $\neq 0$ indicates a problem within the peak-find itself; $B A D \neq 0$ indicates that the estimated mode parameters fail some post-processing criterion
} 


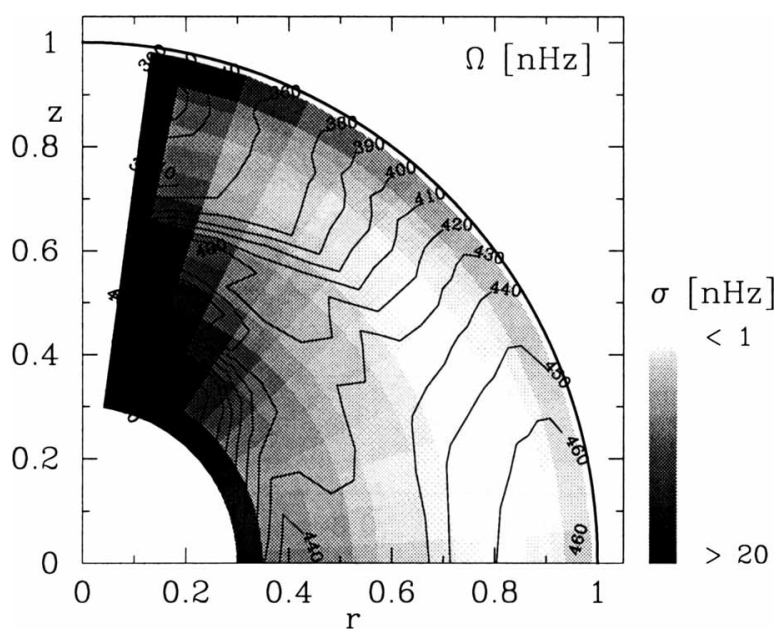

Figure 1. Inversion of GONG project frequencies using $1 \otimes 1$ SOLA (Aarhus group). Contours of isorotation are shown, superimposed on a grey-scale plot of the formal errors. A very dark background means a less reliable determination.

\section{Results}

Figure 1 shows the solar rotation profile inferred from individual $m$ splittings using a $1 \otimes 1$ SOLA inversion. Similar results are shown by Sekii (these proceedings). Results from two other methods - RLS and 2D SOLA - applied to these same data are illustrated in Fig. 2. All of the inversions show the same overall behaviour: the persistence of surface-like differential rotation through much of the convection zone; enhanced rotation around $r=0.95 R$; a transition to essentially latitudinally-independent rotation beneath the convection zone. The inversions compared in Fig. 2 are in quite good agreement, even though the RLS used individual $m$ splittings and the 2D SOLA used $a$ coefficients only up to $a_{7}$. The small but systematic differences in the convection zone (in particular at $60^{\circ}$ latitude) need to be investigated but probably can be understood in terms of the differences in averaging kernels: specifically, the RLS kernels have structure near the surface, so that the interior solution may be biased by the near-surface rotation rate. The averaging kernels differ even more in the deeper interior, where the present data constrain the rotation rate rather poorly. The large asymmetric horizontal bars on the high-latitude SOLA inversion at around $0.4 R$ indicate that the method failed to localize a kernel at this target location.

The sensitivity to different peak-bagging reductions applied to the same 


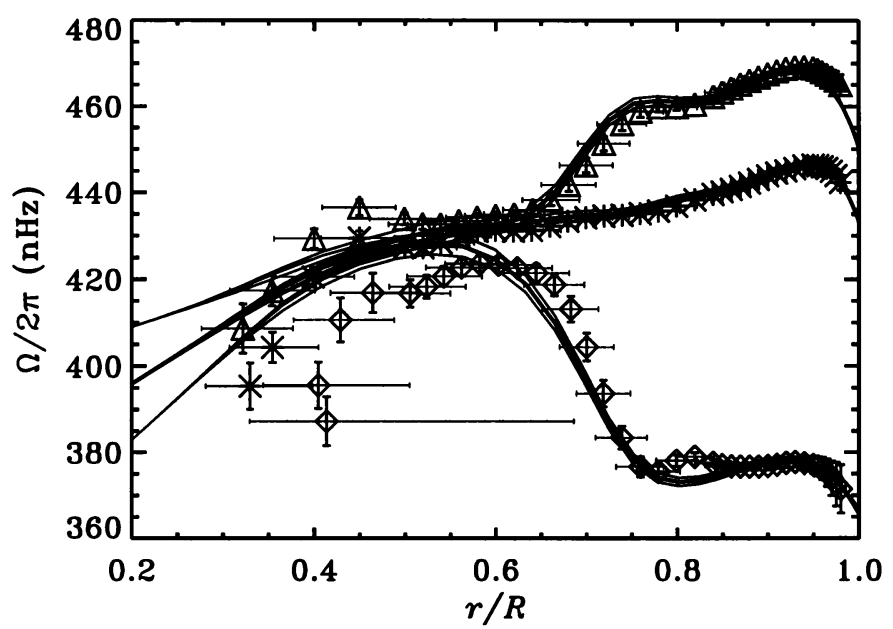

Figure 2. Inversions of GONG project frequencies using 2D RLS (solid curves; QMW group) and 2D SOLA (symbols; Aarhus group) at latitudes $0^{\circ}, 30^{\circ}, 60^{\circ} . \pm 1-\sigma$ formal errors are indicated by extra curves and by vertical error bars on the symbols; horizontal bars represent radial resolution of the averaging kernels.

GONG power spectra is illustrated in Fig. 3, where the results of applying the same 1.5D RLS inversion to $a$ coefficients from the GONG project and from Sylvain Korzennik are compared. The two panels show similar comparisons with similar methods, but from two groups of collaborators. The inferred differences from the two data reductions are slight in the convection zone and outer radiative interior. Only in the deep interior do the two datasets produce strikingly different results (panel a), with the project data indicating a slow rotation while the Korzennik data favour a roughly uniform rotation profile. Because the rotation in the deep interior is poorly constrained, the solution in $r<0.4 R$ is largely a result of extrapolation according to the regularization used. For this reason, in the inversion in panel $\mathrm{b}$, conditions of zero radial and latitudinal gradient have been imposed on the solution at $r=0.3 R$, which effectively enforces a uniform rotation in the deep interior. While this may avoid giving a misleading impression caused by a wild extrapolation in the core, it too is an a priori prejudice and in this case serves to mask the discrepancy between the two datasets.

To investigate the effect of inverting data from different experiments, we have compared inversions of GONG project frequencies with inversions of splittings derived from two-year averaged LOWL data $(5 \leq l \leq 95)$. Results of a 1.5D RLS inversion of both datasets are shown in Fig. 4 . The 

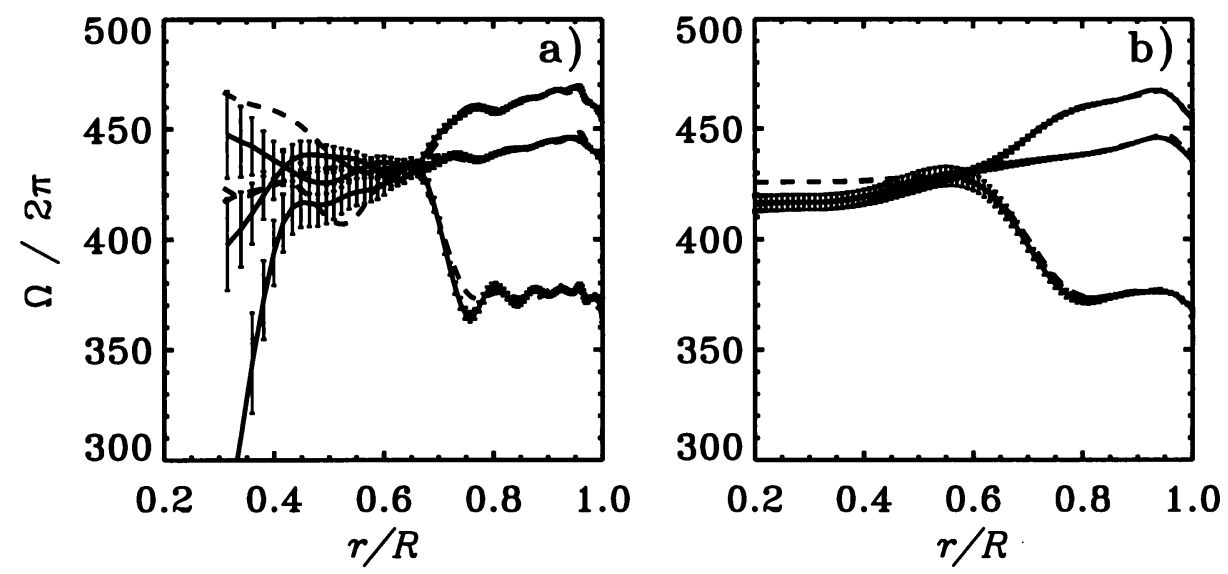

Figure 3. Comparison of inferences from project (continuous curves, with error bars) and SGK $a$ coefficients (broken curves) using 1.5D RLS. The two panels show (a) results from CfA group; (b) results from TIFR group. The solution is depicted at latitudes $0^{\circ}$ (equator), $30^{\circ}$ and $60^{\circ}$.

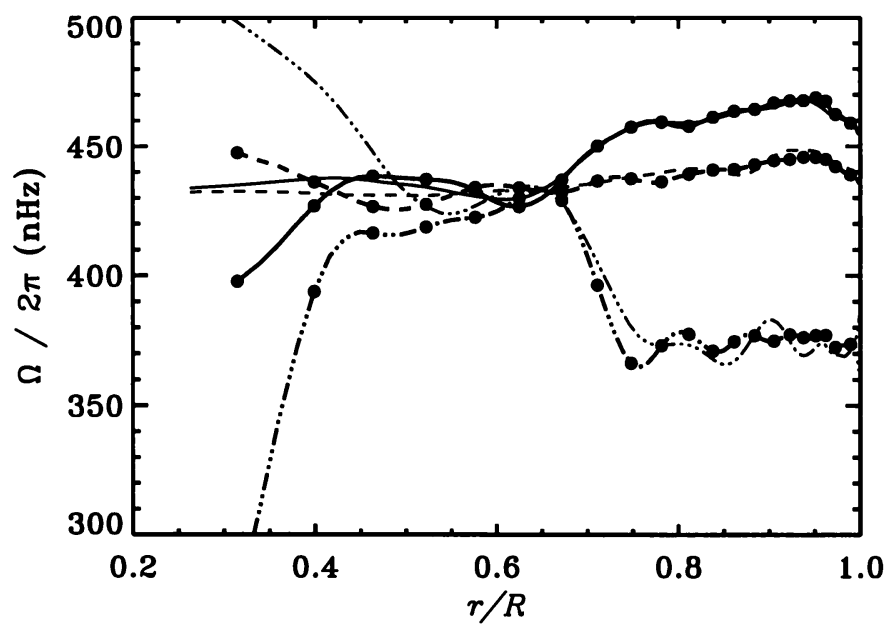

Figure 4. Comparison of inferences from GONG project frequencies and from LOWL frequencies, using a 1.5D RLS inversion (CfA group). The curves with solid dots are the GONG results (as in Fig. 3a). The solution is shown for latitudes $0^{\circ}$ (solid curves), $30^{\circ}$ (dashed) and $60^{\circ}$ (triple-dot dashed). 
main difference is beneath $r=0.5 R$ : comparing with Fig. 3a, it is clear that the differences would have been much smaller in this region if the Korzennik peak-bagging of the GONG data had been used instead of the GONG project frequencies. Given that the LOWL frequencies were determined by J. Schou using an independent peak-bagging procedure (Schou 1992), this could be an indication that the project procedure is causing a systematic error in the inferred rotation beneath $0.5 R$. Aside from that, the most obvious differences between the LOWL and GONG inversions are in the convection zone at $60^{\circ}$ latitude: beneath $0.8 R$ these would again have been reduced by comparing instead with Korzennik GONG frequencies, while the oscillations in the LOWL solution above $0.8 R$ are probably an indication that the inversion parameters need to be chosen to give a little more smoothing in this region.

To summarize our results on the sensitivity of our inferences to changing the observational dataset, the peak-bagging method and the inversion technique, all three lead to some generally small but noticeable differences in the solutions for the internal rotation. Judging from our findings, the uncertainties from all of these are similar in magnitude. In the deep interior (say beneath $0.4 R$ ) the solutions are more poorly constrained by the data and the differences are larger.

A new measurement of some interest to solar dynamo theorists and those interested in the dynamics of the solar interior is the thickness of the tachocline (the layer at the base of the convection zone where the rotation speed changes rather abruptly). According to the analysis of Spiegel \& Zahn (1992), the thickness is determined by the turbulent Prandtl number, i.e. the ratio of the horizontal viscous diffusion coefficient to the (radiative) coefficient of thermal diffusion. The inversion procedures have only a finite resolution, which means that the tachocline may be much thinner than is at first sight suggested by the fairly gradual transitions evident in the inferred rotation profiles (Figs. 2-4). Indeed, if the regularization is modified so that a discontinuity at $0.7 R$ is not penalized, the solution of a $2 \mathrm{D}$ RLS inversion of GONG data "chooses" to adopt just such a discontinuity (compare Fig. 5a with Fig. 5b). By fitting a simple analytical model profile to BBSO data, Kosovichev (1996) recently deduced a thickness of the tachocline of $(0.09 \pm 0.04) R$. Using a similar model, both in a forward sense and to interpret their inversions, Charbonneau et al. (1996) inferred from LOWL data that the layer is thinner, probably no more than $0.06 R$, with a hint also that the tachocline is prolate. By making a nonlinear leastsquares fit to the GONG data of a rotation profile incorporating a transition of adjustable position and width, Sekii (these proceedings) infers that the tachocline is centred on $r=0.696 R$ and has width $0.064 R$. This value is not very different from that considered by Spiegel \& Zahn. It is interesting 

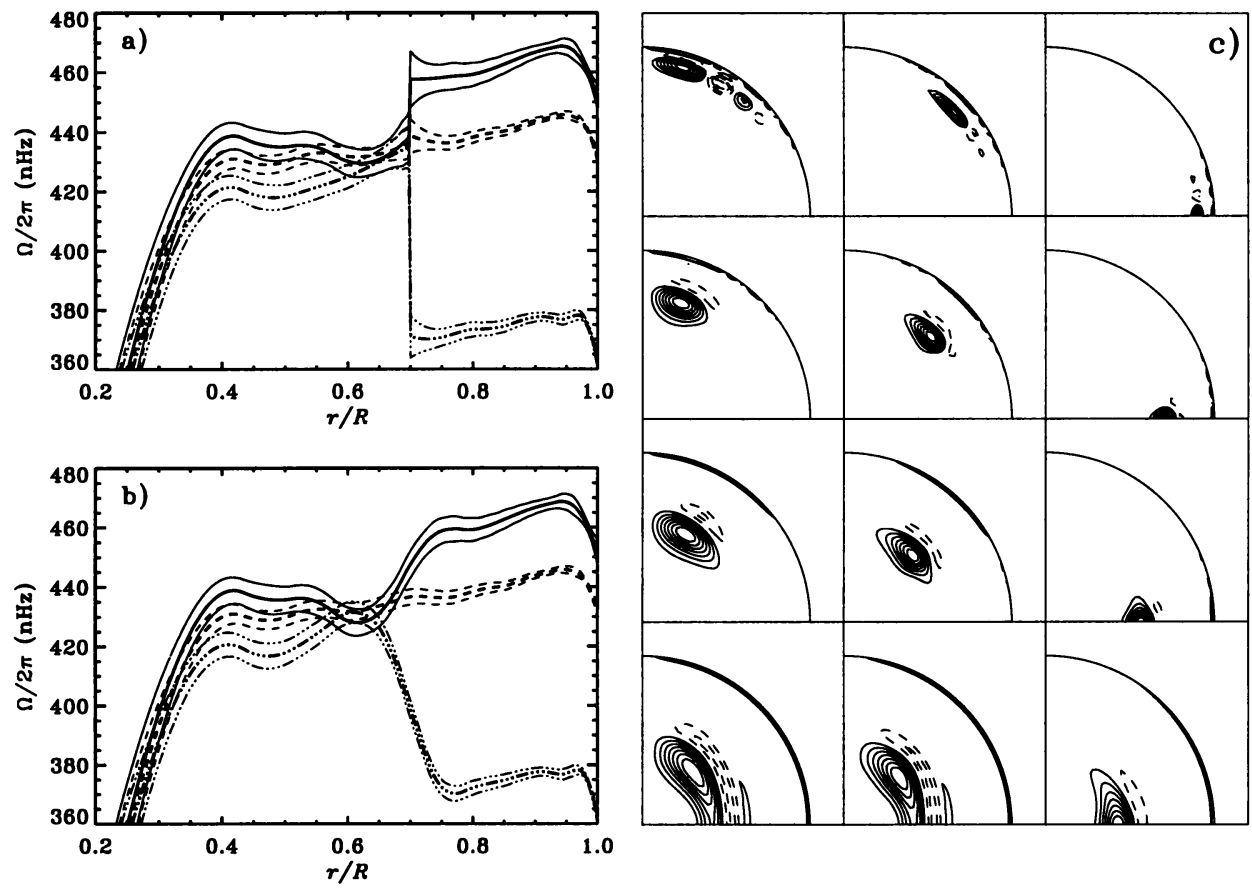

Figure 5. (a) Solution at latitudes $0^{\circ}$ (solid), $30^{\circ}$ (dashed) and $60^{\circ}$ (triple-dot dashed) for a 2D RLS of GONG data, allowing the solution to adopt a discontinuity at the base of the convection zone. Adjacent curves indicate $\pm 1-\sigma$ error limits. (b) As (a) but not allowing a discontinuity ('standard' 2D RLS). (c) Averaging kernels corresponding to the solution in panel $\mathrm{b}$, at target latitudes (left to right) $90^{\circ}, 45^{\circ}$ and $0^{\circ}$ and radii (top to bottom) $0.9 R, 0.7 R, 0.55 R, 0.4 R$. (Nice group)

to note also that the value of the almost uniform angular velocity $\Omega_{c}$ in the radiative interior, about 0.94 of the equatorial value $\Omega_{0}$ in the convection zone, is another indication of the stress in the tachocline. The purely horizontal viscous stress based on two-dimensionally isotropic turbulence assumed by Spiegel \& Zahn leads to $\Omega_{c}=0.91 \Omega_{0}$, whereas if the turbulence were isotropic in three dimensions, and large-scale advection were unimportant, $\Omega_{c}$ would be $0.96 \Omega_{0}$ (Gough 1985). The intermediate observed value suggests perhaps that reality lies between these two extremes. It is important to recognise also that perhaps a more radical modification to our theoretical ideas should be entertained, such as transport by anisotropic two-dimensional turbulence or by Lorentz forces.

With only slight differences, all our inversions show that the inferred rotation rates at latitudes $0^{\circ}, 30^{\circ}$ and $60^{\circ}$ converge below the base of the convection zone. How firmly can we therefore say that the rotation in the radiative interior is independent of latitude? To assess this, we inspect av- 
eraging kernels (Fig. 5c). The kernels show how the solution is a weighted average of the true rotation rate, and hence indicate the resolution achieved. At radius $0.7 R$, averaging kernels can be localized at a wide range of latitudes, indicating that latitudinal variation can be well-resolved. At radii as small as $0.4 R$, however, averaging kernels corresponding to different latitudes are very similar to one another and so the inferred values of the rotation at different latitudes are in fact all similar weighted averages of the true rotation: hence it is no surprise that the inferred rotation appears to be independent of latitude, because the inversion has almost no latitudinal resolution at those depths. (Note that at slightly greater radii, the inferred rotation in Fig. 5 b does exhibit some latitudinal variation: this may be caused by data error.) At radius $0.55 R$ it is still possible to localize kernels at different latitudes with the GONG modeset; yet here the solution exhibits no significant latitudinal variation. Thus beneath the tachocline and down to $0.55 R$ we have strong evidence that the surface latitudinal differential rotation has been eliminated and that the rotation rate is independent of latitude. Beneath that point, however, we do not at present have the latitudinal resolution to say from the GONG data whether or not the rotation varies with latitude.

It is encouraging that, to a first approximation, we get similar results for the Sun's internal rotation using different data, different peak-bagging procedures and different inversion methods. However, to address subtler questions we need to understand the differences that arise from all these three sources: these differences are fairly small throughout much of the Sun, but are substantial in the deep interior. As the random errors are reduced and we push our inversions harder, the systematic errors will become even more important. Clearly, more work is still required both in peak-bagging and in the inversions to get better agreement.

\section{References}

Charbonneau, P. et al. (1996) Observational Constraints on the Dynamical Properties of the Shear Layer at the Base of the Solar Convection Zone, in Sounding Solar and Stellar Interiors, Proc. Symposium IAU 181, poster volume, in press

Gough, D.O. (1985) Theory of solar oscillations, in Future missions in solar, heliospheric and space plasma physics, eds Rolfe, E. \& Battrick, B., ESA SP-235, ESTEC, Noordwijk, $183-197$

Hill, F. et al. (1996) The Solar Acoustic Spectrum and Eigenmode Parameters, Science, 272, 1292

Kosovichev, A.G. (1996) Helioseismic Constraints on the Gradient of Angular Velocity at the Base of the Solar Convection Zone, Astrophys. J. Letters, 469, L61

Schou, J. (1992) On the Analysis of Helioseismic Data, PhD Thesis, Aarhus University.

Spiegel, E.A. \& Zahn, J.-P. (1992) The solar tachocline, Astron. Astrophys., 265, 106

Thompson, M.J. et al. (1996) Differential Rotation and Dynamics of the Solar Interior, Science, 272, 1300 